\title{
OPTIMAL PAIR-WISE SUS ALGORITHM AS PROPORTIONAL FAIR ALGORITHM FOR MU-MIMO-OFDM SYSTEMS
}

\author{
UDC (621.391:621.394.14)
}

\author{
Aleksandra Panajotović, Daniela Milović \\ University of Niš, Faculty of Electronic Engineering, Department of Telecommunications, \\ Niš, Republic of Serbia
}

\begin{abstract}
In this paper, we research proportional fairness of the optimal pair-wise semi-orthogonal user selection (SUS) algorithm used in the MU-MIMO-OFDM system compliant with IEEE 802.11ac standard. Zero-forcing beamforming $(Z F B F)$ is applied in the system as a precoding technique in order to cancel user interferences. Two different scenarios are considered: homogeneous and heterogeneous. Beside the proportional fairness, a throughput performance of the MU-MIMO-OFDM system is analyzed for both scenarios too. Simulation results show that the optimal pair-wise SUS algorithm is an excellent solution to realize the trade-off between the proportional fairness among users and the achieved system throughput.
\end{abstract}

Key words: $M U-M I M O, O F D M$, IEEE 802.11ac, ZFBF, SUS algorithm, FLA algorithm

\section{INTRODUCTION}

There are many reasons why traditional multiple-input multiple-output (MIMO) systems have turned into a new technology known as the multiuser MIMO (MU-MIMO). Some of them are: more users and more devices per user, strong appetite for data, demands in enterprise networks, etc. [1]. Orthogonal frequency-division multiplexing (OFDM) is an effective technique to mitigate the effects of the intersymbol interference (ISI) in a frequency-selective channel by turning a broadband frequency-selective channel into a serial of non-interfering narrow band sub-channels that allow a simple receiver structure to be used [2]. Therefore, the combination of MU-MIMO and OFDM is an attractive solution for WLANs and 4G mobile cellular wireless systems [3]. In order to exploit all benefits that MU-MIMO-OFDM can afford to users, it is required to tackle precoding, resource allocation and user selection.

Received April 2, 2020

Corresponding author: Aleksandra Panajotović

Faculty of Electronic Engineering, Aleksandra Medvedeva 14, 18000 Niš, Republic of Serbia

E-mail: aleksandra.panajotovic@elfak.ni.ac.rs 
Dirty paper coding (DPC) achieves the capacity region of multiple antenna broadcast channel and therefore represents an optimal precoding technique from the realized capacity point of view. However, the implementation of DPC requires significant computational complexity at the receiver and transmitter [4]. On the other hand, linear precoding is a low-complexity but sub-optimal technique which can achieve reasonable performance. Therefore, scientists are focused on investigation of linear precoding techniques such as: zero-forcing beamforming (ZFBF), minimum mean square error (MMSE), successive MMSE, block diagonalization (BD), signal to leakage noise ratiogeneralized eigenvalue decomposition (SLNR-GEVD), etc. [5]. Among them, ZFBF is characterized as very simple precoding technique, without any computational complexity. Moreover, it cancels at the same time both inter- and intra-user interferences [6].

MU-MIMO enables multiple independent radio terminals, with one or more antennas, to access the wireless network at the same time. MU-MIMO exploits the multiuser gain by scheduling multiple users to be able to simultaneously access at the same wireless channel using spatial degrees of freedom offered by MIMO. The optimal scheduling is based on exhaustive search. In the case when number of users, $N_{u}$, is large, it is not an acceptable approach, since the size of the search space, $\sum_{i=1}^{N_{T}}\left(\begin{array}{c}N_{u} \\ i\end{array}\right)$, where $N_{T}$ is the number of transmit antennas, becomes prohibitively large. Consequently, one of challenges in MU-MIMO is a proper user selection. Among many proposed user scheduling algorithms, random (orthogonal) beamforming (RBF) and semi-orthogonal user selection (SUS) are the most popular [7]. The RBF algorithm yields poor performance relative to the SUS algorithm in the practical set of finite users [8], but it does not require a channel state information (CSI) from all users. Moreover, the SUS algorithm in combination with ZFBF gives performance reasonably close to one achieved with DPC [6]. In open technical literature, many SUS-based algorithms have been suggested to be applied in practice: seminal SUS, simplified SUS, generalized multicarrier SUS (GMSUS), pair-wise SUS, optimal pair-wise SUS [6, 9-11].

One of recommendations of the IEEE 802.11ac standard is that scheduled users have to transmit using the same transmission mode (modulation and coding scheme (MCS)) on all subcarriers and all allocated data streams. To this end, the multicarrier MU-MIMO processing at transmitter is complemented with the fast link adaptation (FLA) algorithm. This algorithm can be based on different mapping techniques. The most popular onedimensional mapping techniques, characterized by minor complexity and maximal theoretical improvement, are: uncoded BER $\backslash$ raw BER [12], effective SNR [13], mutual information (MI) [14], PER indicator [15] and mean MI bit mapping (MMIBM) [16].

In this paper we investigate whether the previously proposed optimal pair-wise SUS algorithm [10], which has been shown to provide a good throughput performance for the low and moderate signal-to-noise ratio (SNR) and an excellent performance for high SNR, can be a good solution for a balanced serving of all users in the cell. Therefore, in this paper, beside the homogeneous scenario [10], we analyze the heterogeneous scenario which represents a more realistic case than the homogeneous one. ZFBF is used as precoding technique, while the effective SNR is used as a mapping technique in FLA.

This introduction ends with notational remarks. The vectors and matrices are denoted by lower- and upper-case bold letters, respectively, while scalars are represented with non-bold letters. $(\cdot)^{\mathrm{T}}$ and $(\cdot)^{\mathrm{H}}$ denote the transpose and complex transpose, respectively, $|\mathrm{Q}|$ is the 
cardinality of subset $Q, I_{L}$ is the $L \times L$ identity matrix, $\|a\|$ represents the Euclidian norm of a vector $\boldsymbol{a}$, and $R$ and $C$ are the set of real and complex numbers, respectively.

\section{SYSTEM MODEL}

We consider the downlink of MU-MIMO-OFDM system with $N_{T}$ transmit antennas at the access point (AP) and $N_{u}$ users each equipped with $N_{R}\left(N_{T} \geq N_{R}\right)$ receive antennas. The system operates over $N_{c}$ OFDM subcarriers, out of which $N_{d}$ are used to transmit data while the rest of them correspond to pilots and a guard band. AP serves users with favorable channel condition in certain scheduling period, i.e. AP sends information to subset $Q=\left\{u_{1}, \ldots, u_{|Q|}\right\}, Q \subset N_{u}$, of selected users. $L_{u_{i}}$ spatial streams are allocated to selected user $u_{i}$, and $L_{Q} \triangleq \sum_{i=1}^{|Q|} L_{u_{i}} \leq N_{T}$ has to be satisfied. Let $\mathbf{H}_{u_{i}}[q] \in C^{N_{R} \times N_{T}}$ denote the MIMO propagation channel between AP and $u_{i}$-th user over $q$-th subcarrier. The ZFBF precoding is based on the singular value decomposition (SVD), which applied on $\mathbf{H}_{u_{i}}[q]$ results into

$$
\mathbf{H}_{u_{i}}[q]=\mathbf{U}_{u_{i}}[q] \mathbf{\Sigma}_{u_{i}}[q] \mathbf{V}_{u_{i}}{ }^{H}[q],
$$

where $\mathbf{U}_{u_{i}}[q]=\left[\mathbf{u}_{u_{i}, 1}[q] \ldots \mathbf{u}_{u_{i}, N_{R}}[q]\right] \in C^{N_{R} \times N_{R}}$ and $\mathbf{V}_{u_{i}}[q]=\left[\mathbf{v}_{u_{i}, 1}[q] \ldots \mathbf{v}_{u_{i}, N_{T}}[q]\right] \in C^{N_{T} \times N_{T}}$ are unitary matrices containing the left and the right singular vectors of $\mathbf{H}_{u_{i}}[q]$ and $\Sigma_{u_{i}}[q]=D\left(\sigma_{u_{i}, 1}[q], \ldots, \sigma_{u_{i}, N_{R}}[q]\right) \in R^{N_{R} \times N_{T}}$ is a diagonal matrix whose elements on the main diagonal are singular values of $\mathbf{H}_{u_{i}}[q]$. In order to eliminate inter-user interferences, following post-processing should be applied at the receiver, so now the equivalent channel matrix for $u_{i}$-th user on $q$-th subcarrier is defined as

$$
\tilde{\mathbf{H}}_{u_{i}}[q]=\tilde{\mathbf{U}}^{H}{ }_{u_{i}} \tilde{\mathbf{U}}_{u_{i}}[q] \tilde{\boldsymbol{\Sigma}}_{u_{i}}[q] \tilde{\mathbf{V}}_{u_{i}}{ }^{H}[q]=\tilde{\boldsymbol{\Sigma}}_{u_{i}}[q] \tilde{\mathbf{V}}_{u_{i}}{ }^{H}[q],
$$

where $\tilde{\mathbf{U}}_{u_{i}}[q] \in C^{N_{R} \times L_{u_{i}}}$ contains left singular vectors associated to $L_{u_{i}}$ spatial streams allocated to user $u_{i}$. Similar, $\tilde{\mathbf{V}}_{u_{i}}[q] \in C^{N_{T} \times L_{u_{i}}}$ contains right singular vectors associated to $L_{u_{i}}$ spatial streams and $\tilde{\boldsymbol{\Sigma}}_{u_{i}}[q] \in C^{L_{u_{i}} \times L_{u_{i}}}$ contains singular values.

Inter-user interferences are eliminated with the previously proposed signal postprocessing, while intra-user interferences can be eliminated with the signal pre-processing at the transmitter

$$
\mathbf{x}[q]=\mathbf{W}_{Q}[q] \mathbf{P}_{Q}^{1 / 2}[q] \mathbf{s}_{Q}[q],
$$

with $\mathbf{W}_{Q}[q]=\left[\mathbf{w}_{u_{1}}[q] \ldots \mathbf{w}_{u_{|Q|}}[q]\right] \in C^{N_{T} \times L_{Q}}$ representing the ZFBF precoding matrix on the subcarrier $q$ defined as $\mathbf{W}_{Q}[q]=\tilde{\mathbf{H}}_{Q}^{H}[q]\left(\tilde{\mathbf{H}}_{Q}[q] \tilde{\mathbf{H}}_{Q}^{H}[q]\right)^{-1}$ where $\tilde{\mathbf{H}}_{Q}[q]=\left[\tilde{\mathbf{H}}_{{ }^{\prime}{ }_{1}}^{T}[q] \ldots \tilde{\mathbf{H}}_{{ }{ }_{Q \mid}}^{T}[q]\right]^{T}$ $\in C^{L_{Q} \times N_{T}} . \mathbf{P}_{Q}[q]=\left[\mathbf{P}_{u_{1}}[q] \ldots \mathbf{P}_{u_{Q \mid}}[q]\right] \in C^{L_{Q} \times L_{Q}}$ is the power allocation matrix and $\mathbf{s}_{Q}[q]=$ $\left[\mathbf{s}_{u_{1}}^{T}[q] \ldots \mathbf{s}_{u_{Q}}^{T}[q]\right]^{T} \in C^{L_{Q} \times 1}$ is the vector consists of the information symbols sent to selected users. In the end, the selected user $u_{i}$ disposes with the following signal 


$$
\mathbf{y}_{u_{i}}[q]=\mathbf{P}_{u_{i}}^{1 / 2}[q] \mathbf{s}_{u_{i}}[q]+\tilde{\boldsymbol{\eta}}_{u_{i}}[q],
$$

where $\tilde{\boldsymbol{\eta}}_{u_{i}}[q]$ is a zero-mean circularly symmetric complex Gaussian vector.

\section{OPTIMAL PAIR-WISE SUS ALGORITHM}

The design of the scheduling algorithm is an important issue in MU-MIMO systems. In [17], authors point out that the complexity of pair-wise SUS algorithm is smaller than its traditional counterpart [6]. Therefore, in [10] we have proposed an optimal pair-wise SUS algorithm, applicable in MU-MIMO, which combines both the pair-wise SUS algorithm and exhaustive search. The steps of the optimal pair-wise SUS algorithm are [18]:

Step 1: Initialization

$$
\begin{aligned}
& i=1, \\
& Q_{0}=1, \ldots, N_{u} N_{R},
\end{aligned}
$$

Step 2: Determine the degrees of orthogonality, $\beta_{l, p}$, for this algorithm, between all virtual user pairs $l \neq p$ (each spatial stream is treated as a virtual user).

$$
\beta_{l, p}=\sum_{q=1}^{N_{d}} \frac{\left|\tilde{\mathbf{h}}_{l}[q] \tilde{\mathbf{h}}_{p}^{H}[q]\right|}{\left\|\tilde{\mathbf{h}}_{l}[q]\right\| \tilde{\mathbf{h}}_{p}[q] \|},
$$

where $\tilde{\mathbf{h}}_{l}[q]$ is the equivalent channel gain vector corresponding to the $l$-th spatial stream.

Step 3: Find the pair $P_{i}$, from $Q_{i-1}$, with the smallest degree of orthogonality

$$
P_{i}=\{l, p\}=\arg \max _{l, p \in Q_{i-1}} \beta_{l, p} .
$$

Step 4: Select $i$-th virtual user to be eliminated as follows

$$
\begin{aligned}
& \pi(i)=\arg \min _{r \in P_{i}} \sum_{q=1}^{N_{d}}\left\|\tilde{\mathbf{h}}_{r}[q]\right\|, \\
& Q_{i}=\left\{m \in Q_{i-1} \mid m \neq \pi(i)\right\}, \\
& i \leftarrow i+1,
\end{aligned}
$$

If $Q_{i-1}>N_{T}$, go to Step 3 .

Step 5: Apply the exhaustive search, i.e. calculate the realized throughput for all combination of virtual users selected in Step 4 and find the combination providing maximal throughput, i.e. $Q=\arg \max _{S \subset Q_{i-1}} T(S)$.

\section{FLA ALGORITHM}

In this section, we explain the effective SNR mapping technique used in the FLA algorithm to allocate the appropriate MCS mode to the user which should be served. Namely, once the group of users, $Q$, and their corresponding number of spatial streams, 
$L_{u_{i}}, i=\overline{1,|Q|}$, are selected using some of scheduling algorithms, the adaptive modulation and coding algorithm have to be conducted to provide a better throughput system performance [19]. The MIMO-OFDM channel is time-varying and frequency-selective, so the received signal level varies over time, frequency and spatial streams. So, electing the adequate combination of modulation and coding scheme, i.e. adequate MCS mode, $m$, is a very important issue in improving the throughput of a selected user satisfying at the same time some predetermined quality of service (QoS). That QoS constraint is usually in the form of outage probability of a specified target packet error rate, $\mathrm{PER}_{0}$. The aim of the FLA algorithm is to predict PER for different MCSs from a defined finite set, $M$, and select the best one from both, QoS and maximized throughput point of view.

Since resulting PER depends on many parameters (packet length, allocated MCS, signal-to-interference-plus-noise ratio, etc.), it is a cumbersome task to derive analytical expression for PER [20]. To overcome this challenge all parameters are mapping into the single link quality metric (LQM) associated to a PER value by means of a look-up table obtained either by simulation or real hardware measurements. The effective SNR is one of LQMs which can be found in the open technical literature.

The average PER can be approximated in terms of the average bit error rate (BER) [13] as

$$
\operatorname{PER}\left(m, L, \gamma_{u_{i}}\right) \approx 1-\left(1-\frac{B E R\left(m, L_{r}, \gamma_{u_{i}}\right)}{d_{f}}\right)^{\frac{L}{R_{m}}},
$$

where $L$ is the packet length in number of information bits, $L_{r}$ is the reference packet length, $\boldsymbol{\gamma}_{u_{i}}$ is a vector containing SNRs, over each of $N_{d}$ subcarriers, corresponding to all spatial streams, $L_{u_{i}}$, allocated to the selected user $u_{i}, R_{m}$ is the coding rate, $d_{f}$ is the free distance and $m$ is the MCS value allocated to the selected user $u_{i}$. To evaluate BER depending on $m, L_{r}$ and $\gamma_{i}$, it is necessary to map all these parameters into one known as LQM, in this paper effective SNR, and use look-up table. Effective SNR can be evaluated as

$$
\gamma_{\text {eff }}\left(m, L_{r}, \gamma_{u_{i}}\right)=\alpha\left(m, L_{r}\right) J^{-1}\left(\frac{1}{N_{d} L_{u_{i}}} \sum_{q=1}^{N_{d}} \sum_{j=1}^{L_{u_{i}}} J\left(\frac{\gamma_{v_{j}}^{u_{i}}[q]}{\alpha\left(m, L_{r}\right)}\right)\right),
$$

where $\gamma_{v_{j}}^{u_{i}}[q]$ is the received SNR for $j$-th stream allocated to user $u_{i}$ on subcarrier $q$, $\alpha\left(m, L_{r}\right)$ is parameter allowing the model to be adapted to both the packet length and the specific characteristic of the corresponding MCS and $J(\cdot)$ is a model-specific LQM function [16], and in case of an effective SNR it is defined as $J(x)=\exp (-x)$ [13].

Mapping between the effective SNR and BER can be described with the following equation

$$
\operatorname{BER}\left(m, L_{r}, \gamma_{u_{i}}\right) \approx B E R^{A W G N}\left(m, L_{r}, \gamma_{e f f}\left(m, L_{r}, \gamma_{u_{i}}\right)\right)
$$

where $B E R^{A W G N}\left(m, L_{r}, \gamma\right)$ is the curve characterizing BER achieved when system uses MCS $m$ to transmit packet length $L_{r}$ across the AWGN channel with average SNR $\gamma$.

Optimal MCS allocated to the selected user $u_{i}$ can be derived as 


$$
m_{u_{i}}^{o p t}=\underset{m \in M}{\arg \max }\left(1-\frac{B E R^{A W G N}\left(m, L_{r}, \gamma_{e f f}\right)}{d_{f}}\right)^{\frac{L}{R_{m}}}
$$

subject to

$$
\gamma_{e f f}\left(m, L_{r}, \gamma_{u_{i}}\right) \geq \Gamma\left(m, L_{r}, P_{o u t}\right)
$$

with $\Gamma\left(m, L_{r}, P_{\text {out }}\right)$ representing a threshold value obtained as the value of $\gamma_{\text {eff }}\left(m, L_{r}, \gamma_{u_{i}}\right)$ for which the constrain condition

$$
\mathrm{P}_{r}\left\{B E R^{A W G N}\left(m, L_{r}, \gamma_{e f f}\left(m, L_{r}, \gamma_{u_{i}}\right)\right)>B E R_{o}\left(m, L_{r}\right)\right\} \leq P_{\text {out }},
$$

is satisfied with equality.

\section{NUMERICAL RESULTS}

In order to investigate the fairness of the optimal pair-wise SUS scheduling algorithm, this section presents the simulation results obtained using parameters from the IEEE $802.11 \mathrm{ac}$ standard. The system operates at $5.25 \mathrm{GHz}$ carrier frequency with the bandwidth of $20 \mathrm{MHz}$ that is divided into $N_{c}=64$ subcarriers out of which $N_{d}=52$ are used to carry data while the rest correspond to pilot signals and guard intervals. The AP has $N_{T}=4$ transmit antennas, while all users are equipped with the $N_{R}=2$ receive antennas. A spacetime-frequency-selective fading channel is presented through $\mathrm{B}$ and $\mathrm{E}$ channel profile [21]. The values of parameters for FLA are taken from Table I in [11]. In this paper, unlike the work in [10] where a homogenous scenario is considered, in which all users sit on circumference centered at AP and all experience the same average SNR, a more realistic scenario is analyzed. It is a heterogeneous scenario in which users are at different distances from the AP, and therefore each of them is exposed to a different level of SNR.

One of the most relevant fairness indicators is the Jain's fairness index (JFI) [22]

$$
J F I=\frac{\left[\sum_{i=1}^{N_{u}} R_{u_{i}}(t)\right]^{2}}{N_{u} \sum_{i=1}^{N_{u}} R_{u_{i}}^{2}(t)},
$$

where $R_{u_{i}}(t)$ is the date rate of user $u_{i}$. JFI ranges from $1 / N_{u}$ (only one user is served) to 1 (all users are served at the same rate). Figures 1 and 2 depict per-user throughput JFI versus different number of users and for different schedule algorithms. Two different channel profiles (Fig. 1 - B channel, Fig. 2 - E channel) are considered. The B channel characterizes environment with little-to-moderate frequency selectivity (small offices and houses), while the $\mathrm{E}$ channel characterizes an environment with a moderate-to-large frequency selectivity (large indoor spaces such as airports or sport halls). 


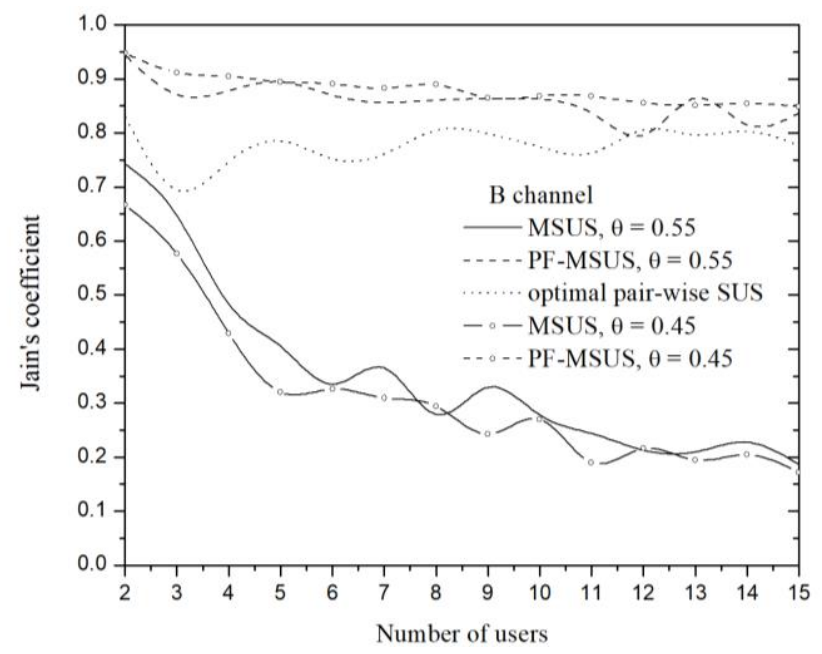

Fig. 1 Jain's fairness index comparison for individual user's throughput for different scheduling algorithms for heterogeneous scenario as a function of the number of users for B channel.

Regardless of the applied environment and degree of orthogonality $(\theta)$ related to MSUS and PF-MSUS algorithms [11. Eq. (9)], the PF-MSUS algorithm leads to a rather high JFI value ( $>0.8$ for $\mathrm{B}$ channel, $>0.7$ for $\mathrm{E}$ channel). An increase of the number of users does not provoke a decrease of JFI, indicating in that way a large degree of fairness which this algorithm provides. In contrast, under MSUS, JFI decreases rapidly from $\sim 0.7$ for $N_{u}=2$ to $\sim 0.2$ for $N_{u}=15$ for the B channel, and from $\sim 0.5$ to $\sim 0.1$ for the E channel. Let us explain that. The large number of users increases probability that one (or maybe a few) user is located very close to AP, so its channel condition favors automatically it to be served by AP with MSUS scheduling algorithm. Namely, MSUS algorithm does not take into account that selected user has been already served in previous scheduling periods, causing unfairness between users. Therefore, Jain's fairness index is low for large number of users in the cell for MSUS algorithm, which was. expected [11]. It is also expected that the PF-MSUS algorithm would show a worse throughput performance than the MSUS algorithm [11], and it is confirmed through Fig. 3. So, a trade-off between the fairness and the realized throughput is inevitable, when we speak about these two algorithms. Our previous results [10] have shown that the MU-MIMO system with the optimal pair-wise algorithm has provided a better throughput performance than the system with the MSUS algorithm for a homogenous scenario. Results from Figs. 1 and 2 point out the additional advantage of the optimal pair-wise SUS algorithm, besides the realized throughput which is justified in heterogeneous scenario too (Fig. 3). It can be classified into the group of proportional fair algorithms. Its JFI does not decline with an increase in the number of users. its value is rather high and takes values in range between 0.7 and 0.85 for the $\mathrm{B}$ channel and between 0.6 and 0.7 for the E channel. A slightly smaller value of JFI for the E channel can be explained with the frequency selectivity which remains in OFDM sub-channels for such type of environment. 


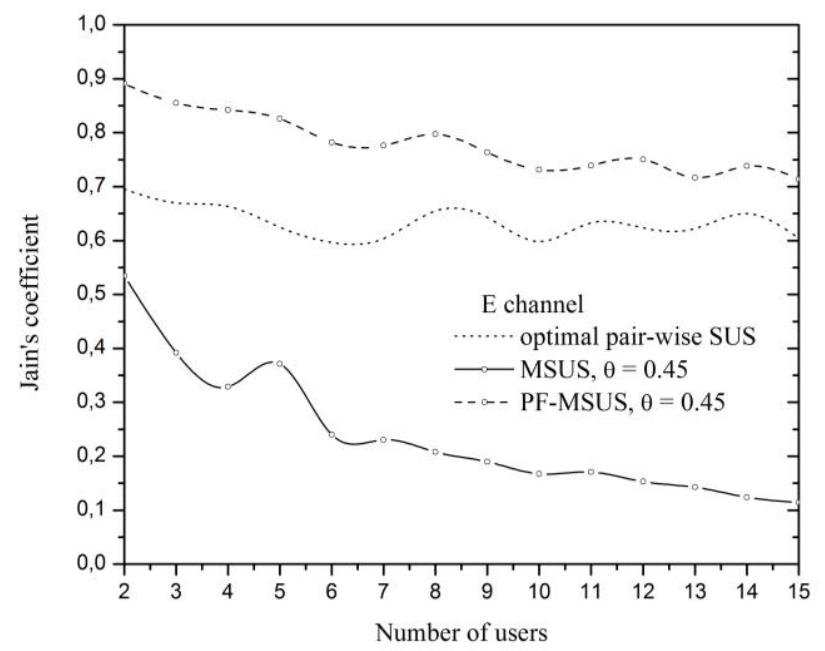

Fig. 2 Jain's fairness index comparison for individual user's throughput for different scheduling algorithms for heterogeneous scenario as a function of the number of users for E channel.

Let us analyze results presented in Fig. 3. Firstly, these results for MSUS and PF-MSUS algorithms are totally in accordance with our previous published results [11]. Secondly, in case of a random distribution of users over circular area (heterogeneous scenario) the optimal pair-wise SUS scheduling algorithm still provides selection of the user group which provides the higher throughput than MSUS and PF-MSUS algorithms. That conclusion stays valid for any number of users which should be served by AP and for any power value which AP transmits. If we compare results proposed in Fig. 3 with ones in Fig. 1 from [10], we can recap that in real scenario, that is heterogeneous, the optimal pair-wise SUS algorithm

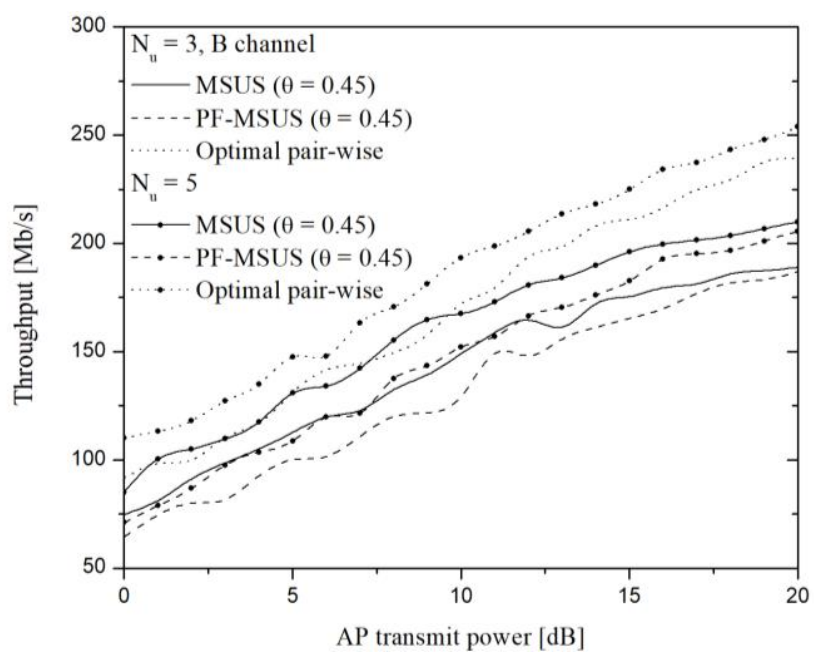

Fig. 3 Throughput comparison for different scheduling algorithms for heterogeneous scenario for $N_{u}=3$ and $N_{u}=5$. 
provides the best throughput performance of MU-MIMO system over all range of average SNR, even better than in the homogenous scenario.

Having in mind all presented results and observation from this Section, we can make a conclusion that from both points of view, the proportional fairness and realized throughput, the optimal pair-wise algorithm can be addressed as the best option for practical realization over all considered SUS-based algorithms.

\section{CONCLUSION}

This work has tackled the problem of proportional fairness of the scheduling algorithm applicable in the MU-MIMO-OFDM system compliant with IEEE 802.11ac. In this aim, we have analyzed the MU-MIMO-OFDM system with the ZFBF precoding technique, applied to eliminate inter- and intra-user interferences, while the FLA algorithm has been used to realize as much as possible higher throughput satisfying at the same time predetermined QoS and IEEE 802.11ac recommendations.

Excellent throughput performance, confirmed in our previous papers dealing with a homogenous scenario, has been once more justified for MU-MIMO system using the optimal pair-wise SUS algorithm, in the realistic - heterogeneous scenario. The main contribution of this paper is the proof of the proportional fairness of the optimal pair-wise SUS algorithm, which favours it as one of endorsed algorithms to be used..

Acknowledgement: This work has been supported by the Ministry of Education, Science and Technological Development of the Republic of Serbia.

\section{REFERENCES}

[1] Qaulcomm Atheros, Inc, “802.11ac MU-MIMO: Bridging the MIMO gap in Wi-Fi”, January, 2015. Available: www.qualcomm.com

[2] R. van Nee, R. Prassad, OFDM for Wireless Multimedia Communications, Artech House, 2000.

[3] M. Esslaoui, F. R. -Palou, G. Femenias, "Opportunistic multiuser MIMO for OFDM network" in Proceedings of $8^{\text {th }}$ International Workshop on Multi-Carrier Systems \& Solution, Herrsching, Germany, pp. 1-5, 2011. Available: http://dx.doi.org/10.1109/MC-SS.2011.5910709

[4] J. Lee, N. Jindal, "Dirty paper coding vs. linear precoding for MIMO broadcast channels" in Proceedings of Fortieth Asilomar Conference on Signals, Systems and Computers, Pacific Grove, CA, USA, pp. 1-5, 2007. Available: http://dx.doi.org/10.1109/ACSSC.2006.354855

[5] M. A. Martin, Developments in Wireless Network Prototyping, Design and Deployment: Future Generations, Information Science Reference, 2012.

[6] T. Yoo, A. Goldsmith, "On the optimality of multiantenna broadcast scheduling using zero-forcing beamforming," IEEE Journal of Selected Areas in Communications, vol. 24, no. 3, pp. 528-541, 2006. Available: http://dx.doi.org/10.1109/JSAC.2005.862421

[7] H. - C. Yang, M. -S. Alouini, Order Statistics in Wireless Communications, Cambridge University Press, 2011.

[8] G. Lee, Y. Sung, "A new approach to user scheduling in massive multi-user MIMO broadcast channels," IEEE Transactions on Communications, vol. 66, no. 4, pp. 1481-1495, 2018. Available: http://dx.doi. org/10.1109/TCOMM.2017.2786670

[9] J. Mao, J. Gao, Y. Liu, G. Xie, "Simplified semi-orthogonal user selection for MU-MIMO systems with ZFBF," IEEE Wireless Communication Letters, vol. 1, no. 1, pp. 42-45, 2012. Available: http://dx.doi. org/10.1109/WCL.2012.010912.110119

[10] A. Panajotović, N. Sekulović, D. Milović, "Optimal pair-wise SUS scheduling algorithm for multiuser MIMO” in Proceedings of Conference ICEST 2018, Sozopol, Bulgaria, pp. 179-182, 2018. 
[11] A. Panajotović, F. R. -Palou, G. Femenias, "Adaptive uniform channel decomposition in MU-MIMOOFDM: Application to IEEE 802.11ac," IEEE Transactions on Wireless Communications, vol. 14, no. 5, pp. 2896-2910, 2015. Available: http://dx.doi.org/10.1109/TWC.2015.2396513

[12] M. Lamarca, F. Rey, "Indicators for PER prediction in wireless systems: a comparative study" in Proceedings of IEEE $61^{\text {st }}$ Vehicular Technology Conference, Stockholm, Sweden, 2005. Available: http://dx.doi.org/10.1109/VETECS.2005.1543413

[13] G. Martorell, F. R. -Palou, G. Femenias, "Cross-layer fast link adaptation for MIMO-OFDM based WLAN," Wireless Personal Communications, vol. 56, no. 3, pp. 599-605, 2011. Available: http://doi. org/10.1007/s11277-010-9992-9

[14] K. Sayan, J. Zhung, K. Stewart, "Link performance abstraction based on mean mutual information per bit (MMIB) of the LLR channel", https://www.semanticscholar.org/paper/Link-PerformanceAbstraction-based-on-Mean-Mutual-Sayana-Zhuang/c1e19aa7f74b0178602ac2f9ae3d0c87a24949e3

[15] M. Lampe, T. Giebel, H. Rohling, W. Zirwas, "PER prediction for PHY mode selection in OFDM communication systems" in Proceedings of IEEE Global Telecommunications Conference, San Francisco, USA, 2003. Available: http://dx.doi.org/10.1109/GLOCOM.2003.1258196

[16] T. L. Jensen, S. Kant, W. Joachim, B. H. Fleury, "Fast link adaptation for MIMO OFDM," IEEE Transactions on Vehicular Technlogy, vol. 59, no. 8, pp. 3766-3778, 2010. Available: http://dx.doi.org/ 10.1109/TVT.2010.2053727

[17] S. Dierks, N. Jünger, "Scheduling for massive MIMO with excess antennas" in Proceedings of International ITG Workshop on Smart Antennas, Munich, Germany, 2016. Available: https://ieeexplore.ieee.org/document/ 7499100

[18] A. Panajotović, N. Sekulović, D. Milović, "Optimal pair-wise SUS scheduling algorithm in adaptive UCD-based MU-MIMO-OFDM" in Proceedings of Conference INDEL 2018, Banja Luka, Bosnia and Hercegovina, 2018.

[19] J. Cavers, "Variable-rate transmission for Rayleigh fading channels," IEEE Transactions on Communications, vol. COM-20, no. 1, pp. 15-22, 1972. Available: http://dx.doi.org/10.1109/TCOM.1972.1091106

[20] S. Simoens, S. Rouquette-Léveil, P. Sartori, Y. Blakenship, B. Classon, "Error prediction for adaptive modulation and coding in multiple-antenna OFDM systems," Signal Processing-Special Section: Advances in Signal Processing-assisted Cross-layer Designs, vol. 86, no. 8, pp. 1911-1919, 2006. Available: https://doi.org/10.1016/j.sigpro.2005.09.033

[21] J. Kermoal, J. Schumacher, K. I. Pedersen, P. E. Mongensen, F. Fredeiksen, "A stochastic MIMO radio channel model with experimental validation," IEEE Journal of Selected Areas in Communications, vol. 20, no. 6, pp. 1211-1226, 2002. Available: http://dx.doi.org/10.1109/JSAC.2002.801223

[22] R. Jain, D. M. Chiu, W. R. Hawe, "A quantitative measure of fairness and discrimination for resource allocation in shared computer systems," Digital Equipment Corporation. Eastern Research Laboratory, DEC-TR-301, 1984. Available: https://arxiv.org/abs/cs/9809099 